\title{
Intricate treatment of Endophthalmitis
}

\author{
Ionela losub*, Livia Sram \\ Department of vitreo-retinal surgery, Eye Emergency Hospital, Bucharest, Romania
}

\begin{abstract}
Uveitis implies an inflammation of the uveal tract. However, the term is commonly used to describe many forms of intraocular inflammation involving not only the uvea but also the retina and its vessels. We present the case of a 9 year-old girl, hospitalized in Emergency Eye Hospital of Bucharest, for uveitis with hypopyon. Special investigations (infectious, rheumatological, imaging, immunological) revealed endophthalmitis of unknown etiology. The patient received a complex treatment, comprising of systemically administered tienam and prednisone, and of local treatment and intravitreal with triamcinolone injection. Given the complications of the cataract in the course of the disease, vitrectomy and Extra-Capsular Extraction (EEC) with intraocular lens implantation followed. The functional outcome was very good, periodic monitoring is required to be prolonged.

Keywords: endophthalmitis, intravitreal injection, cataract
\end{abstract}

\section{Introduction}

Endophthalmitis implies inflammation, often purulent, involving all the intraocular tissues, except the sclera. Endophthalmitis includes anterior and posterior uveitis. Anterior uveitis is the most common form, accounting for $3 / 4$ of cases. Presentation is typically with sudden onset of unilateral pain, photophobia and redness. Visual acuity is usually good at presentation except in eyes with severe hypopyon [1].

Miosis due to sphincter spasm may predispose to the formation of posterior synechiae. Aqueous cells indicate disease activity and their number reflects disease severity.

Hypopyon is a feature of intense inflammation in which cells settle in the inferior part of the anterior chamber and form a horizontal level.

Received: August 2014; Accepted after review: September 2014; Published: September 2014

*Corresponding author: Ionela losub, MD, Department of vitreo-retinal surgery, Eye Emergency Hospital, Bucharest, Romania. Email: geanina iosub@yahoo.com
Posterior uveitis encompasses retinitis, choroiditis and retinal vasculitis. Active lesions are characterized by whitish retinal opacities with indistinct borders due to surrounding edema [2].

\section{Case report}

We report the case of a 9 year-old girl presenting with blurred vision, photophobia of the right eye for 5 days, without any history of ocular trauma. External examination revealed right eye with good perception of hand movements, right eye intraocular pressure of $12 \mathrm{mmHg}$, right eye normal ductions and versions movement.

Slit lamp examination indicated cellular deposits on the corneal endothelium, hypopyon, fibrinous exudate. Fundus examination was unexplorable.

The results of the laboratory investigations for inflammatory syndrome are presented in Table 1. The serological tests were negative for Toxocara canis and Toxoplasma gondii (Table 2). 
Table 1. Special investigations: inflammatory syndrome

\begin{tabular}{lcl}
\hline Investigation & Patient value & Normal value \\
\hline WBC & 13,5 & $3,9-9,6 / 1000 / \mathrm{uL}$ \\
NEUTROPHILS & 84,3 & $37,0-73,0 / \%$ \\
LYMPHOCYTES & 11,8 & $20-55 / \%$ \\
FIBRINOGEN & 457 & $180-350 \mathrm{mg} / \mathrm{dL}$ \\
VSH & 60 & $5-15$ \\
\hline
\end{tabular}

Table 2. Special investigations: infectious syndrome

\begin{tabular}{lll}
\hline Investigation & Patient value & Normal value \\
\hline Toxocara canis & $3,901 \mathrm{NTU}$ & $<9=$ Negative \\
Toxoplasma gondii & $<0,13 \mathrm{Ul} / \mathrm{ml}$ & $<1=$ Negative \\
\hline
\end{tabular}

Imagistic investigations: Ocular ultrasound shows right eye with multiple inflammatory vitreous echoes and choroidal edema (Figure 1). Computer tomography was performed to exclude sarcoidosis, multiple sclerosis and primary intraocular lymphoma (Figure 2).

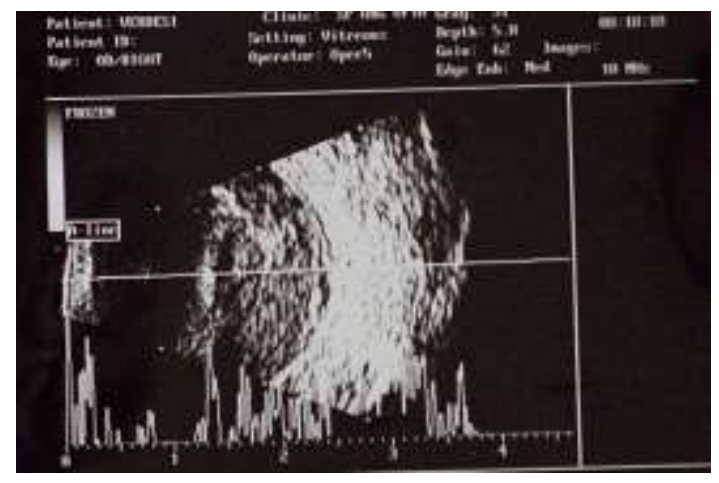

Fig. 1. Ocular ultrasound

The diagnosis is right eye endophthalmitis sustained by anterior uveitis: keratic precipitates, hypopyon, fibrinous exudate and posterior uveitis: vitreous haze, choroidal edema.

Differential diagnosis includes idiopathic endophthalmitis or systemic associations: parasitic (toxoplasmosis, toxocariasis), bacterial (tuberculosis, syphilis, Lyme disease, brucellosis, endogenous bacterial endophthalmitis, cat-scratch disease), viral (CMV retinitis, HIV microangiopathy), fungal (fungal infection has not been found), neurological (multiple sclerosis, Vogt-Koyanagi-Harada syndrome, Behçet syndrome), rheumatic (spondyloarthropathies, juvenile arthritis), gastro-intestinal (Crohn disease, Whipple disease), sarcoidosis.

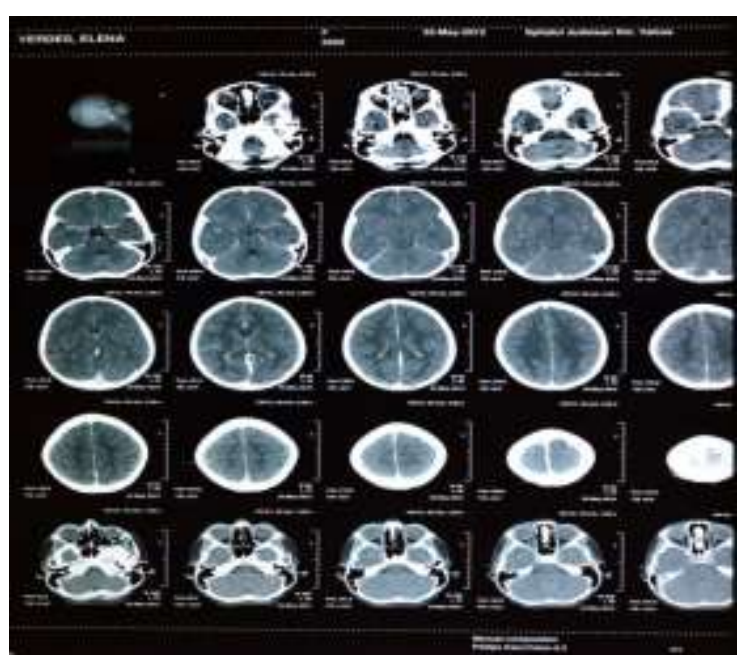

Fig. 2. Skull CT

The treatment was systemic with Tienam (imipenem and cilastatin) $500 \mathrm{mg}$ i.v., every 12 hours, PDN $30 \mathrm{mg} /$ day (after 7 days $25 \mathrm{mg}$ ), Diflucan (fluconazolum) $1 \mathrm{cp} /$ day, Dicarbocalm (calcium carbonate, magnesium carbonate and trisilicate) 1 tbx3/day.

The local treatment included: Netildex (neomycin sulfate and dexamethasone), 
Edicin (vancomycinum), Axetine (cefuroximum), Diflucan (fluconazolum), Tropicamid (tripicamide), Fenefrin (phenylephrinum) (1 dx6/day) and intravitreal triamcinolone (TA) injection (Figure 3).

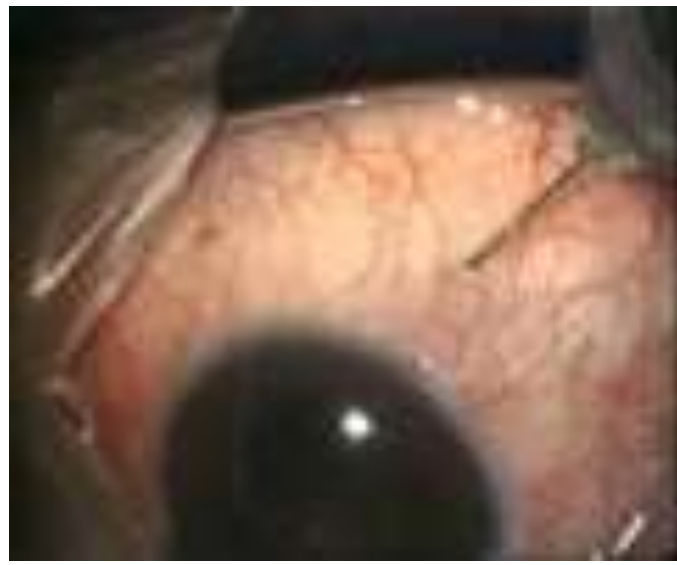

Fig. 3. Intravitreal injection

After treatment, the visual acuity of the patient improved from 0.04 to 0.1 and $0.2 \mathrm{~m}$, but after one month, the visual acuity decreased to counter finger at $0.2 \mathrm{~m}$ because of the cataract complications that occurred (Figure 4).

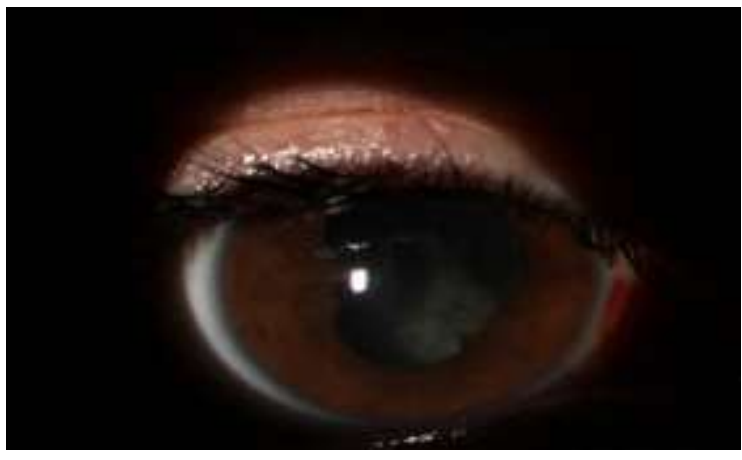

Fig. 4. Posterior subcapsular cataract

Vitrectomy and EEC with intraocular lens implantation followed. The functional outcome was very good. Discharge vision was 1/6, $1 / 4$ pinhole. After a month vision was $0.8-0.9$ with glasses (+0.5 sfd -0.75 cyld $/ 180$ degrees). Titmus test: fly, $\mathrm{ABC}+$, Lang test+, Worth test+.

The functional prognosis is excellent with a final visual acuity of $0.2 \mathrm{~m}$. The general prognosis is favorable considering the sideeffects of systemic medication (prednisone), which also requires a prolonged monitoring of the patient (Figure 5).

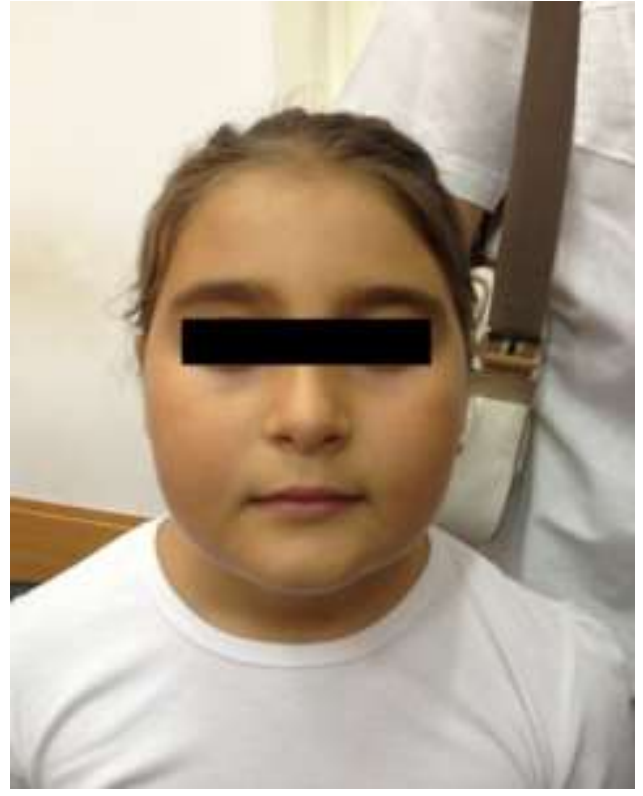

Fig. 5. Cushingoid syndrome, post-prednisone therapy

Treatment in uveitis comprises of predominantly the use of anti-inflammatory and antibiotic therapy [3]. It is important to keep in mind that drugs used for treating uveitis have potential side-effects and this should always be weighed against the decision to treat. Also, it must be emphasized that the use of systemic therapy should be carried out in conjunction with a physician who is competent to deal with the associated complications both to the underlying disease and to the therapy $[4,5]$.

\section{Conclusions}

In conclusion, this clinical case is particular because it is an idiopathic endophthalmitis. It required exhaustive interclinical examinations. Treatment was complex and included medical (tienam, the most "powerful" antibiotic; prednisone, the "strongest" anti-inflammatory) and invasive treatment: the intravitreal injection of TA and the vitrectomy. Another feature of the case is the secondary cataract, solved by lens phacoemulsification and IOL$\mathrm{CP}$ implantation. The case requires extensive monitoring, being an idiopathic disease, relapses may occur. Periodical pediatric examinations are necessary, due to the side effects of medication [6]. 


\section{References}

1. Kansky J, Bowling B. Clinical Ophtalmology - A Systematic Approach, $7^{\text {th }}$ Edition. Philadelphia: Elsevier Saunders, 2011.

2. Krieglstein GK, Weinreb RN. Essential in Ophthalmology - Uveitis and Immunological Disorders. Berlin: Springer Berlin Heidelberg, 2010.

3. Khaw PT, Shah P, Elkington AR. ABC of Eyes, $4^{\text {th }}$ Edition. London: BMJ Publishing Group,
2004.

4. Quillen DA, Blodi BA. Clinical Retina. Chicago: American Medical Association Press, 2002.

5. European Glaucoma Council. Terminology and guidelines for glaucoma - English version, $3^{\text {rd }}$ Edition. Savona: Editrice Dogma, 2008.

6. Field D, Tillotson J. Eye Emergencies: A practitioner's guide. Cumbria, Keswick: M\&K Publishing, 2007. 